\title{
Orthogonal and Least-Squares Based Coordinate Transforms for Optical Alignment Verification in Radiosurgery
}

\author{
Ernesto Gomez $\mathrm{PhD}^{1}$, Yasha Karant $\mathrm{PhD}^{1}$, Veysi Malkoc MS ${ }^{1,2}$, Mahesh R. Neupane ${ }^{1}$, \\ Keith E. Schubert $\mathrm{PhD}^{1}$, Reinhard W. Schulte $\mathrm{MD}^{3}$ \\ ${ }^{1}$ California State University, San Bernardino, California \\ ${ }^{2}$ Ohio State University, Columbus, Ohio \\ ${ }^{3}$ Loma Linda University Medical Center, Loma Linda, California \\ egomez@csci.csusb.edu, ykarant@,csci.csusb.edu \\ mneupane@csci.csusb.edu,schubert@csci.csusb.edu, \\ veysimalkoc.@yahoo.com \\ rschulte@dominion.llumc.edu
}

Keywords: orthogonal transform, least squares, radiosurgery, optical alignment verification

\begin{abstract}
Radiosurgery is a non-invasive treatment technique applying focused radiation beams. It requires high geometric accuracy as misalignment can cause damage to the surrounding healthy tissues and loss of the therapeutic effect. One promising technique to insure submillimeter alignment accuracy of the radiation beam is to optically monitor the position of the beam axis relative to a frame firmly attached to the patient's skull using an optical alignment system. The optical alignment method requires three-dimensional coordinate transforms. This paper compares the standard least squares technique for transforming the coordinate system with an orthogonal transform technique based on the comparison of marker triangles. Orthogonal transforms have good numerical properties and preserve distance, which gives this technique advantages over non-orthogonal techniques. The mathematics behind each technique is covered and alignment results on real test equipment are used to illustrate the differences between the transforms.
\end{abstract}

\section{Introduction}

Radiosurgery is a radiation therapy modality that delivers a high single radiation dose to a well-defined target in the brain [1]. It is usually performed with the Gamma Knife, a linear accelerator delivering megavoltage photon beams (LINAC radiosurgery) or a charged particle accelerator (e.g., proton radiosurgery). The last two methods require an isocentrically rotating gantry and patient positioner in order to place multiple overlapping radiation fields with different orientation on the target.

Any radiosurgery technique demands a very high geometric accuracy since a geometric miss of the target can lead to serious consequences such as loss of tumor control and deleterious side effects caused by radiation necrosis. In LINAC and proton radiosurgery, the required submillimeter accuracy is more difficult to accomplish since it relies on the mechanical stability of a large, rotating gantry (Fig. 1). The isocenter sphere, i.e., a virtual sphere that contains all central beam axes as the gantry completes a 360-degree revolution, typically has a diameter of one to two millimeters [2]. 


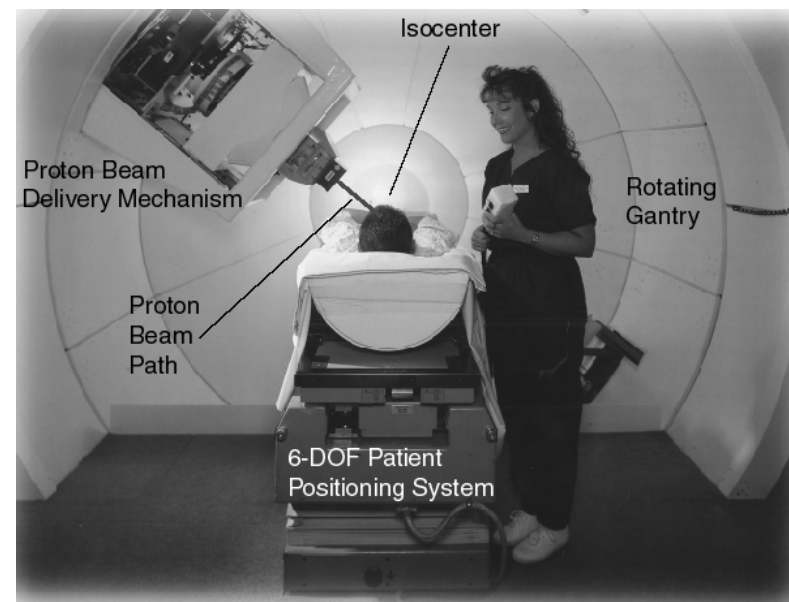

Fig. 1. The proton treatment gantry at Loma Linda University Medical Center. The proton beam rotates isocentrically about the longitudinal patient axis.

One possible solution to assure submillimeter alignment accuracy in gantry-based radiosurgery techniques is to optically monitor the position of the beam axis with respect to the target using visible markers that are attached to both the radiosurgery cone (a device that contains the field-shaping collimator) and the stereotactic frame immobilizing the patient. Online optical monitoring of these markers allows, in principle, to determine the actual position of the beam axis with respect to the anatomical target point, ideally, with submillimeter accuracy. An important part of this monitoring process is to establish accurate coordinate transformations between the local (marker-based) and global (camera-based) coordinate systems.

In this paper, we report on the performance of two types of coordinate transformations used with a prototype camera-based optical alignment system installed in the Proton Treatment Center at Loma Linda University Medical Center (LLUMC). The first coordinate transformation is an orthogonal transformation that preserves the distances between markers; the second transformation is a standard leastsquare based coordinate transformation that does not necessarily preserve geometric distances. Both transformations were applied to compute the distance of a phantom marker with known stereotactic coordinates from the central axis of an expanded laser beam, representing the collimated radiation beam.

\section{Materials and Methods}

\subsection{System Components}

In the following, we describe the components of the prototype optical alignment system that has been developed at LLUMC for applications in proton radiosurgery and is currently in its non-clinical test phase.

2.1.1. Camera System. The Camera System consists of three high-resolution CCD cameras (Vicon Motion Systems, Inc., Oxford, UK), which are placed in an equilateral triangle configuration at the edges of the circular disk at the back of gantry (Fig. 1), $1.62 \mathrm{~m}$ from the isocenter (Fig. 2). The cameras have a focal length of $25 \mathrm{~mm}$, and are strobed with infrared light, being sensitive only to the reflective markers of the marker systems (see below). The focus of each camera is directed to the center between the marker systems attached to the patient and the radiosurgery-cone. Since the back of the gantry and the proton beam delivery cone rotate as one unit, the position of the cone relative to the cameras is fixed except for a small deviation due to the sag of the gantry, which is seen by the cameras. The camera configuration is shown below.

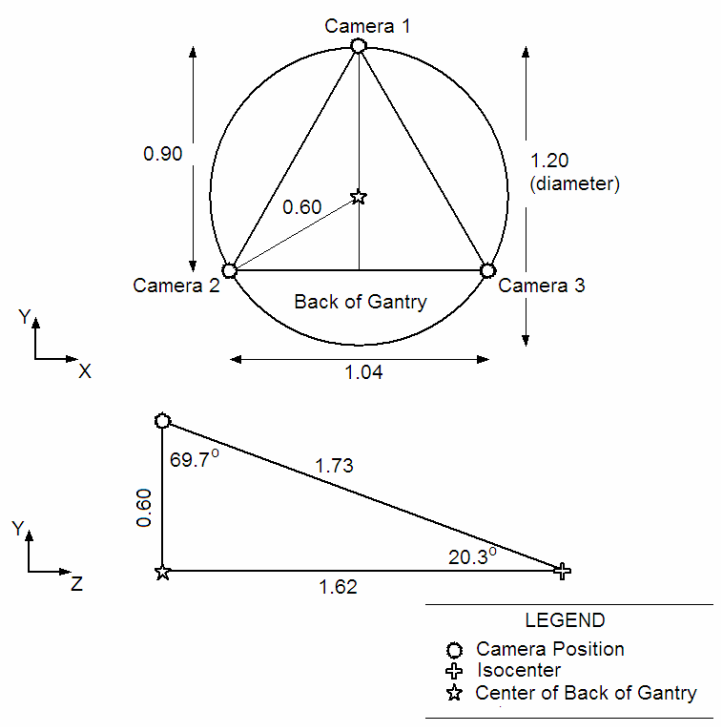

Fig. 2. Geometrical arrangement of the Camera System for monitoring patient and beam position in the proton gantry at LLUMC (dimensions are in meters).

This camera configuration maximizes the visibility of both the patient-centered and the beam-centered marker systems. In the typical clinical setup, the cameras point at the top of the patient's head.

The Vicon camera system requires a static followed by a dynamic calibration. The static calibration is performed with an L-shaped arrangement of 2D reflective markers, while the dynamic calibration involves periodic movements of a wand with two spherical reflective markers arranged at a distance of $10.0 \mathrm{~cm}$ in front of the cameras. 
2.1.1. Marker Systems. A marker system for tracking a patient's head position was developed to accurately monitor the position of the anatomical radiosurgery target with respect to the proton beam. The markers consist of 5-mm ceramic spheres, which are covered by retro-reflective tape (Vicon Motion Systems, Inc., Oxford, UK). The patient-centered marker system is attached to a Leksell $G$ stereotactic frame (Electa Instruments, $\mathrm{AB}$, Stockholm, Sweden), which will be firmly affixed to the patient's skull during the treatment procedure. The marker system consists of 23 markers distributed around the head, minimizing the interference with the proton beam (Fig. 3). The redundancy of markers permits at least six markers seen by all three cameras through the entire motion range of the patient positioning system. The location of each marker in the stereotactic coordinate system of the Leksell $G$ frame was measured by a certified dimensional inspection laboratory (Dimensional Metrology Laboratory, Riverside, CA, USA) to an accuracy of better than $+/-0.1 \mathrm{~mm}$.

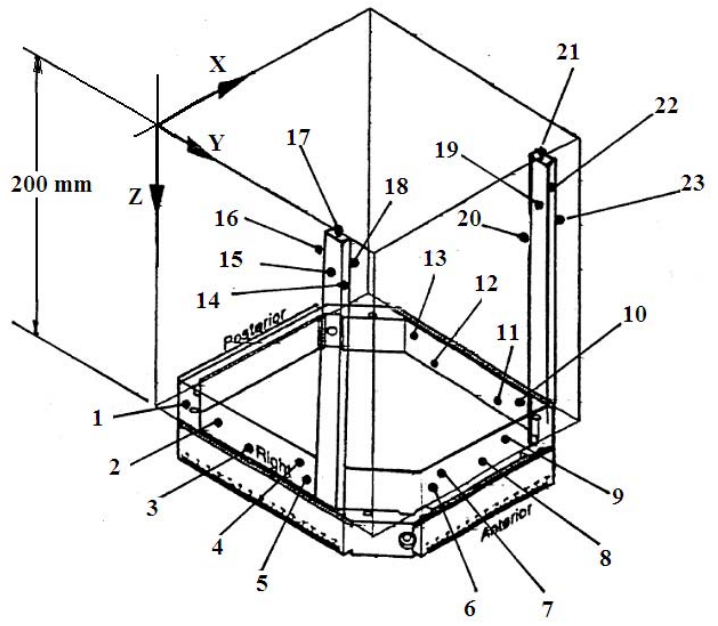

Fig. 3. Patient-centered marker system attached to the Leksell $G$ frame marking the stereotactic reference system. The markers are arranged so at least 6 markers can be seen simultaneously by all three cameras and the access of the proton beam to the patient's head is minimally restricted.

The beam-centered marker system is attached to the radiosurgery treatment cone, which delivers a collimated proton beam to the patient. It consists of nine reflective markers, identical to those used for the patient-centered marker system, which are arranged in the form of a cross (Fig. 4). The position of each marker in the beam-centered reference system was measured to an accuracy of better than $+/-0.1 \mathrm{~mm}$ by the same laboratory.

\subsection{Verification Procedure}

To verify the performance of the optical alignment system, a laser expander fitting tightly inside the treatment cone was used to simulate a circular proton beam of $1-\mathrm{cm}$ diameter. The laser beam was optically centered on a simulated target, thus providing a nearperfect target alignment with a submillimeter residual error. The actual position of the target and the central beam axis was then calculated based on registration of the patient- and beam-centered marker systems with the camera system. The shortest distance between calculated target and the beam axis provided the actual alignment registration error.

The platform for the simulated anatomical target was provided by a metal phantom base that attaches reproducibly to the stereotactic frame (Fig. 5). Spherical ceramic markers of $3-\mathrm{mm}$ diameter were attached to rods of three different lengths that can be placed in any of five base holes, thus providing welldefined simulated target locations in 15 possible stereotactic positions.
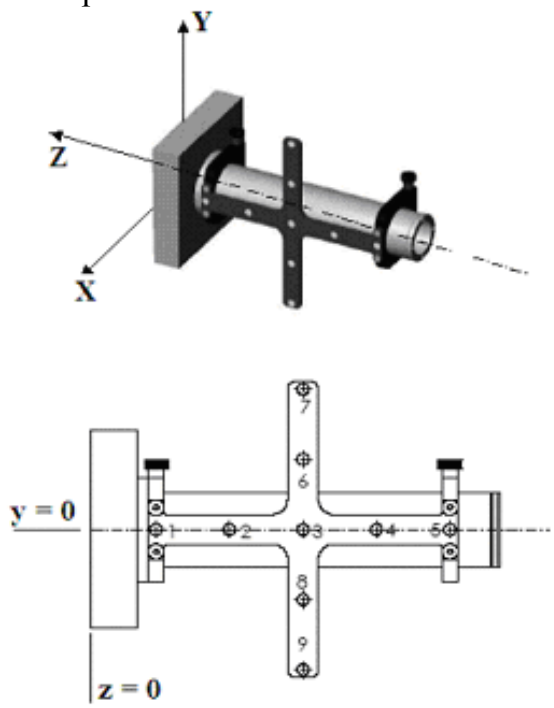

Fig. 4. Beam-centered marker system attached to the radiosurgery treatment cone. The cross-shaped marker arrangement is facing the three cameras and is providing information on the actual beam position.

The location of stereotactic marker position in the Leksell $\mathrm{G}$ frame coordinate system was measured by the dimensional inspection laboratory to an accuracy of better than $+/-0.1 \mathrm{~mm}$.

A single phantom base marker was attached to the phantom base and optically aligned with the laser beam, which was placed to the side of the stereotactic frame. Any residual offset between marker center and beam center was corrected by the patient positioning system (PPS) to which the stereotactic frame was 
attached. The PPS allows fine movements along three orthogonal axes in steps of $50 \mu \mathrm{m}$.

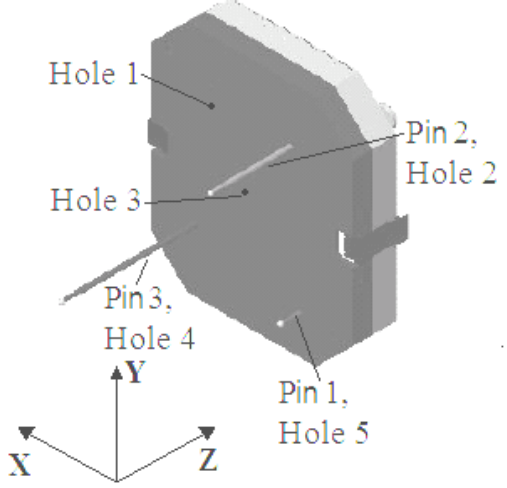

Fig. 5. Phantom base used to verify the accuracy of the optical alignment system. Spherical markers attached to rods of three different lengths can be placed in any of five different phantom holes providing 15 different marker locations.

Once the phantom marker was aligned to the laser beam axis to within $+/-0.1 \mathrm{~mm}$, the location of the patient- and beam-centered marker systems was captured during a static trial of the Vicon camera system. The coordinates in the global Vicon system were exported as ASCII text files and analyzed with orthogonal and least-square-based coordinate transformation programs written in Mathcad 11 (Mathsoft Engineering and Education, Inc., Cambridge, MA, USA). The programs transformed the beam axis from beam-centered coordinate system to the patient-centered coordinate system and calculated the distances between the beam axis and the simulated target based on the known stereotactic target position. Since, the target had been nearly perfectly aligned with the laser beam, any residual distance between phantom marker and beam axis was interpreted as the actual alignment registration error of the optical alignment system.

\subsection{Coordinate Transformations}

2.3.1 Orthogonal Transformation. In the following, the superscript $(g)$ indicates global coordinates and the superscript $(l)$ indicates local coordinates. In general, the coordinates of at least three markers in the local (patient- or beam-centered) reference system will also be known in the global (camera-based) reference system. All coordinate systems considered here are right-handed. The geometric interpretation of the coordinate transform between both systems is shown in Fig. 6.

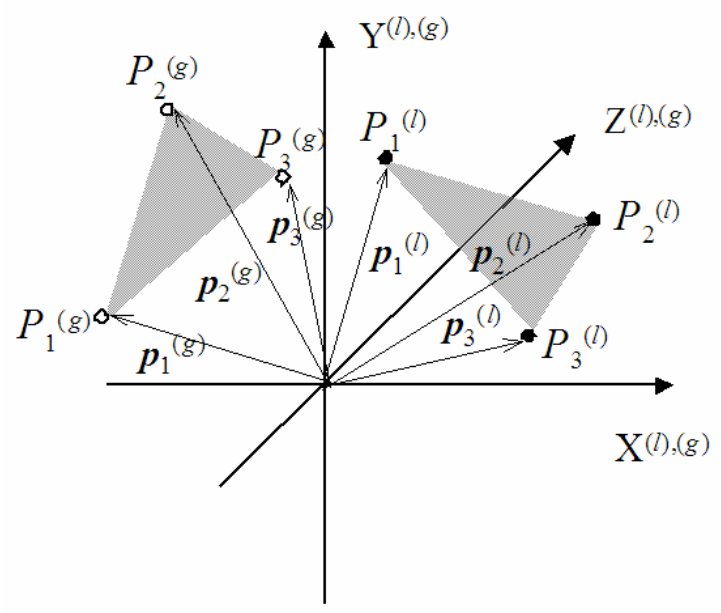

Fig. 6. Conceptual arrangement of marker triangles in two reference systems. The goal of the coordinate transformation is to make one triangle coplanar and collinear to the second.

Consider the triangle $P_{1}^{(1)}, P_{2}^{(1)}, P_{3}^{(1)}$ in the local coordinate system, which is formed by the three known markers (Fig. 6). Let $\boldsymbol{p}_{1}{ }^{(1)}, \boldsymbol{p}_{2}{ }^{(1)}$, and $\boldsymbol{p}_{3}{ }^{(1)}$, denote the position vectors pointing from the origin of the local reference system to the central point of each marker. Note that lower-case bold letters are used here to denote vectors, and upper-case bold letters to denote matrices. The corresponding position vectors to the triangle $\mathrm{P}_{1}{ }^{(\mathrm{g})}, \mathrm{P}_{2}{ }^{(\mathrm{g})}, \mathrm{P}_{3}{ }^{(\mathrm{g})}$ in the global reference system are called $\boldsymbol{p}_{1}{ }^{(g)}, \boldsymbol{p}_{2}{ }^{(g)}$, and $\boldsymbol{p}_{3}{ }^{(g)}$. One may obtain the clearest perception of the rotations and translation involved in the coordinate transformation between the two reference system by assuming that the origins and axes of both coordinate systems coincide, and that the vectors $\boldsymbol{p}_{1}{ }^{(l)}, \boldsymbol{p}_{2}{ }^{(l)}, \boldsymbol{p}_{3}{ }^{(l)}$ and $\boldsymbol{p}_{1}{ }^{(g)}, \boldsymbol{p}_{2}{ }^{(g)}, \boldsymbol{p}_{3}{ }^{(g)}$ represent two different marker sets (Fig. 6). Then, the task to find a coordinate transformation between the two coordinate systems is identical to finding the transformation that maps the local marker set onto the global marker set.

In general, the orthogonal transformation equation that maps corresponding local points onto global points, can be expressed as follows:

$$
\boldsymbol{p}_{\boldsymbol{n}}{ }^{(\mathrm{g})}=\boldsymbol{M}_{\boldsymbol{B}} \cdot \boldsymbol{M}_{\boldsymbol{A}} \cdot \boldsymbol{p}_{\boldsymbol{n}}{ }^{(l)}+\boldsymbol{t}(n=1-3)
$$

where $\boldsymbol{M}_{\boldsymbol{A}}$ and $\boldsymbol{M}_{\boldsymbol{B}}$ are $3 \times 3$ matrices representing proper rotations. The matrix $\boldsymbol{M}_{\boldsymbol{A}}$ corresponds to a rotation that makes the triangle formed by the local marker set coplanar with the plane formed by the global marker set. The matrix $\boldsymbol{M}_{\boldsymbol{B}}$ corresponds to an "in-plane" rotation, which aligns two corresponding triangle sides with respect to each other. After performing these two rotations on the local triangle, the vector $\boldsymbol{t}$ corrects for the residual translational difference between the two point sets. 
Expressions for the matrices $\mathbf{M}_{\mathbf{A}}$ and $\mathbf{M}_{\mathbf{B}}$ have been derived previously by Weaver et al. [3]. They obtained the following expression for the matrix $\boldsymbol{M}_{\boldsymbol{A}}$ :

$$
\boldsymbol{M}_{A}=\left(\begin{array}{ccc}
a+o_{A 1}^{2}(1-a) & n_{A 3}+o_{A 1} o_{A 2}(1-a) & -n_{A 2}+o_{A 1} o_{A 3}(1-a) \\
-n_{A 3}+o_{A 2} o_{A 1}(1-a) & a+o_{A 2}^{2}(1-a) & n_{A 1}+o_{A 2} o_{A 3}(1-a) \\
n_{A 2}+o_{A 3} o_{A 1}(1-a) & -n_{A 1}+o_{A 3} o_{A 2}(1-a) & a+o_{A 3}^{2}(1-a)
\end{array}\right)
$$

where $\boldsymbol{n}_{\boldsymbol{A}}=\boldsymbol{n}^{(l)} \times \boldsymbol{n}^{(\mathrm{g})}$ with $\boldsymbol{n}^{(l)}=\left(\boldsymbol{p}_{3}{ }^{(l)}-\boldsymbol{p}_{1}{ }^{(l)}\right) \times\left(\boldsymbol{p}_{2}{ }^{(l)}-\right.$ $\left.\boldsymbol{p}_{1}{ }^{(l)}\right)$ and $\boldsymbol{n}^{(g)}=\left(\boldsymbol{p}_{3}{ }^{(g)}-\boldsymbol{p}_{1}{ }^{(g)}\right) \times\left(\boldsymbol{p}_{2}{ }^{(g)}-\boldsymbol{p}_{1}{ }^{(g)}\right), a=\cos (\alpha)$ $=\boldsymbol{n}^{(l)} \cdot \boldsymbol{n}^{(g)}$, and $\boldsymbol{o}_{\boldsymbol{A}}=\boldsymbol{n}_{\boldsymbol{A}} / \sin (\alpha)$.

Multiplication of the local position vectors $\boldsymbol{p}_{1}{ }_{1}^{(l)}, \boldsymbol{p}_{2}{ }_{2}^{(l)}$, and $\boldsymbol{p}_{3}{ }^{(l)}$ by matrix the $\boldsymbol{M}_{\boldsymbol{A}}$ yields new vectors $\boldsymbol{p}_{1}{ }_{1}^{(l)}$, $\boldsymbol{p}_{2}{ }_{2}^{(l)}$, and $\boldsymbol{p}_{3}{ }_{3}{ }^{(l)}$ which form a triangle that is now coplanar but not collinear with the global marker triangle. The matrix $\boldsymbol{M}_{\boldsymbol{B}}$ can be expressed as

$$
\boldsymbol{M}_{\boldsymbol{B}}=\left(\begin{array}{ccc}
b+o_{B 1}^{2}(1-b) & n_{B 3}+o_{B 1} o_{B 2}(1-b) & -n_{B 2}+o_{B 1} o_{B 3}(1-b) \\
-n_{B 3}+o_{B 2} o_{B 1}(1-a) & b+o_{B 2}^{2}(1-b) & n_{B 1}+o_{B 2} o_{B 3}(1-b) \\
n_{B 2}+o_{B 3} o_{B 1}(1-b) & -n_{B 1}+o_{B 3} o_{B 2}(1-b) & b+o_{B 3}^{2}(1-b)
\end{array}\right)
$$

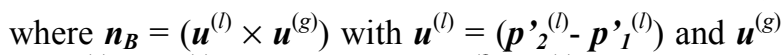
$=\left(\boldsymbol{p}_{2}{ }^{(g)}-\boldsymbol{p}_{1}{ }^{(g)}\right), b=\cos (\beta)=\boldsymbol{u}^{(l)} \cdot \boldsymbol{u}^{(g)}$, and $\boldsymbol{o}_{\boldsymbol{B}}=\boldsymbol{n}_{\boldsymbol{B}}$ $/ \sin (\beta)$.

Multiplication of the local position vectors first by matrix $\boldsymbol{M}_{\boldsymbol{A}}$ and then by matrix $\boldsymbol{M}_{\boldsymbol{B}}$ yields new vectors $\boldsymbol{p}{ }_{1}{ }_{1}^{(l)}, \boldsymbol{p},{ }_{2}^{(l)}$, and $\boldsymbol{p},{ }_{3}^{(l)}$. The triangle described by these vectors is now coplanar and collinear with the triangle formed by the global positioning vectors. Finally we translate $\boldsymbol{p},{ }_{\boldsymbol{I}}^{\left({ }^{()}\right)}$into $\boldsymbol{p}_{\boldsymbol{I}}{ }^{(\mathrm{g})}$ by adding the vector $\boldsymbol{t}=\boldsymbol{p}_{\boldsymbol{I}}{ }^{(\mathrm{g})}$ $p,{ }_{1}^{(l)}$. If no systematic or random errors are involved, the triangles will now exactly superimpose.

The two rotations involved in the transformation can be combined into one rotation by calculating the matrix $\boldsymbol{M}_{\boldsymbol{A} B}=\boldsymbol{M}_{\boldsymbol{B}} \cdot \boldsymbol{M}_{\boldsymbol{A}}$. We then have

$$
\boldsymbol{v}^{(g)}=\boldsymbol{M}_{\boldsymbol{A B}} \cdot \boldsymbol{v}^{(l)}+\boldsymbol{t}
$$

for transformations of any vector $\boldsymbol{v}$ from the local to the global coordinate system. Since the rotation matrix can be inverted, one can also transform in the opposite direction:

$$
\boldsymbol{v}^{(l)}=\left(\boldsymbol{M}_{\boldsymbol{A B}}\right)^{-1} \cdot\left(\boldsymbol{v}^{(g)}-\boldsymbol{t}\right)
$$

This inverse transformation can be used to transform any vector from the global coordinate system into a local coordinate system.

2.3.1 Least Squares Transformation. The procedure of least squares (LS) minimization was used as an alternative method to establish a coordinate transformation between local and global reference systems. The LS method minimizes the sum of the squared residuals after transformation of all available markers resulting in the most likely estimate of the coefficients of the transformation matrix.

In the last section, we saw that we could express the relationship between the local and global position vectors of the two marker sets by

$$
\begin{aligned}
\boldsymbol{v}^{(g)} & =\boldsymbol{M}_{\boldsymbol{A} \boldsymbol{B}} v^{(l)}+t \\
& =\left[\begin{array}{ll}
\boldsymbol{M}_{\boldsymbol{A} \boldsymbol{B}} & \boldsymbol{t}
\end{array}\right]\left[\begin{array}{c}
\boldsymbol{v}^{(l)} \\
\boldsymbol{1}
\end{array}\right] \\
& \equiv \boldsymbol{X}^{T} \boldsymbol{A}^{T}
\end{aligned}
$$

where $\boldsymbol{A}=\left[\begin{array}{ll}\boldsymbol{v}^{(l) T} & 1\end{array}\right]$, and the superscript $\mathrm{T}$ means transpose. If at least three marker coordinate vectors are known in the local and global reference system, respectively, one can find the LS solution for $\boldsymbol{X}^{T}$ as follows. Let $\boldsymbol{Q}^{(l)} \boldsymbol{R}^{(l)}$ be the QR-factorization of $\boldsymbol{A}$, thus $\boldsymbol{Q}^{(l)}$ is an orthogonal matrix and $\boldsymbol{R}^{(l)}$ is upper triangular. It follows that

$$
\begin{aligned}
\boldsymbol{v}^{(g)} & =\boldsymbol{X}^{T} \boldsymbol{A}^{T} \\
& =\boldsymbol{X}^{T} \boldsymbol{R}^{(l) T} \boldsymbol{Q}^{(l) T} \\
\boldsymbol{v}^{(g)} \boldsymbol{Q}_{l} & =\boldsymbol{X}^{T} \boldsymbol{R}^{(l) T}
\end{aligned}
$$

which can be solved by back substitution to yield the desired transform matrix $\boldsymbol{X}^{T}$ since $\boldsymbol{R}^{(l) T}$ is lower triangular. The main problem with this solution is that the matrix $\boldsymbol{R}$ contains scalings, and the matrix $\boldsymbol{Q}$ can contain reflections as well as rotations. Both can cause alignment errors. In practice the scaling error is usually the larger one.

\section{Results and Discussion}

Figure 7a shows the alignment registration errors of the optical alignment system in the initial 12 independent test runs. The residual errors resulting from the orthogonal transform was one to two orders of magnitude smaller than the LS transform in every test run. The orthogonal transformation yielded an alignment error of $2.8 \pm 2.0 \mathrm{~mm}$ (mean \pm standard deviation under estimation), ranging from 0.5 to 5.5 $\mathrm{mm}$. For the LS transformation the alignment error was $61 \pm 33 \mathrm{~mm}$, ranging from 8.9 to $130 \mathrm{~mm}$. The latter errors are certainly unacceptable for the purpose of radiosurgery alignment verification. 


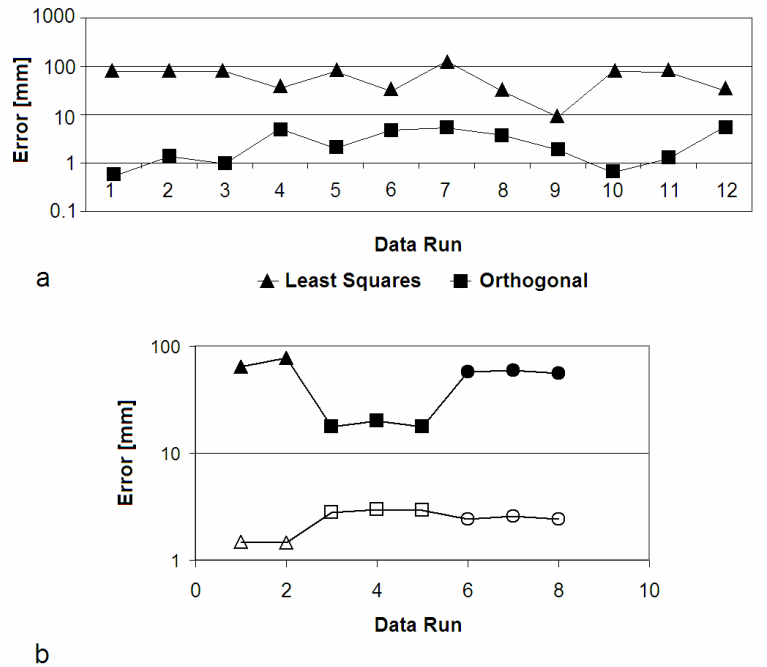

Fig. 7. Alignment error for the two different transforms used to calculate the offset between the phantom base marker and the beam axis. (a) Initial 12 independent experimental runs for the same target position. (b) Additional 8 runs after improving the camera position and calibration technique; open symbols - orthogonal transform, closed symbols - least squares transform, different shapes correspond to different phantom marker positions.

There are two main reasons for the large difference between the two transforms. First, the orthogonal transforms have a norm of one, which means that the accuracy of the camera system itself will be preserved and the errors introduced by the cameras will not appreciably magnified. The second advantage of the orthogonal transform is that it preserves the distances measured. The LS transform we used here involved a non-orthogonal matrix, so it is prone to error magnification and does not preserve the distances in the transformation. Since the Vicon camera system has a potential accuracy of $0.1 \mathrm{~mm}$ and an observed accuracy of about $0.5 \mathrm{~mm}$, it is essential for stereotactic applications to preserve this accuracy.

During our initial tests, we discovered that the quality of the camera calibration as well as optimal camera positioning was key to successful use of the system. While the cameras system, including three cameras, has a theoretical accuracy of $0.1 \mathrm{~mm}$, in practice, we initially experienced an accuracy of 0.3 $1.0 \mathrm{~mm}$ as estimated by comparing camera-measured and known distances of markers in our marker systems.
After improving camera position and calibration technique, additional eight data runs were taken, the results of which are shown in Figure $7 \mathrm{~b}$. The orthogonal transform now showed 1-3 millimeter accuracy, while the reproducibility of independent runs for the same target position was now in the submillimeter range. The orthogonal transform continued to outperform the LS transform by one to two orders of magnitude both in accuracy and reproducibility.

\section{Conclusions}

This work demonstrates that an orthogonal transform has definitive advantages over a nonorthogonal least squares transform. Further possibilities for improvement need to be considered to consistently achieve submillimeter accuracy. These include possibly increasing the number of cameras, as well as optimizing the geometric configuration of the reflective markers. We will continue to work in this direction.

\section{References}

[1] Pollock E. B., Contemporary Stereotactic Radiosurgery: Technique and Evaluation. Futura Pub. Co., Armonk, NY, 2002.

[2] Hartmann G. H., and W. Lutz, Quality Assurance Program on Stereotactic Radiosurgery: Report from a Quality Assurance Task Group. Springer, Berlin, New York, 1995.

[3] K. Weaver, V. Smith, J. D. Lewis, B. Lulu, C.M. Barnett, S.A. Leibel, P. Gutin, D. Larson, and T. Phillips, "A CTbased computerized treatment planning system for I-125 stereotactic brain implants", Int J Radiat Oncol Biol Phys. 18,1990 , pp. $445-54$

\section{Acknowledgements}

We thank the Henry L. Guenther Foundation for generously funding this work. Dr. Michael Moyers provided useful assistance in operating the patient positioner during this research. 